\title{
Immunoglobulin Domain
}

National Cancer Institute

\section{Source}

National Cancer Institute. Immunoglobulin Domain. NCI Thesaurus. Code C13806.

The major histocompatibility complex (MHC) molecules are made of 2 chains, alpha and beta. In class I, the alpha chain is composed of 3 extracellular domains, a transmembrane region and a cytoplasmic tail while the beta chain is of a single extracellular domain. In class II, both the alpha and the beta chains are composed of 2 extracellular domains, a transmembrane region and a cytoplasmic tail. The immunoglobulin (Ig) constant chain domains and a single extracellular domain in each type of $\mathrm{MHC}$ chains are homologous and approximately one hundred amino acids long, and include a conserved intra-domain disulfide bond. Members of the immunoglobulin superfamily are found in hundreds of proteins of different functions, e.g., antibodies, the giant muscle kinase titin and receptor tyrosine kinases. Immunoglobulin-like domains may be involved in protein-protein and protein-ligand interactions. (InterPro IPR007110) 